Article

\title{
The Effects of Inhomogeneous Elasticity and Dislocation on Thermodynamics and the Kinetics of the Spinodal Decomposition of a Fe-Cr System: A Phase-Field Study
}

\author{
Wooseob Shin, Jeonghwan Lee and Kunok Chang * \\ Department of Nuclear Engineering, Kyung Hee University, Yongin-city 17104, Korea; \\ wooseob@khu.ac.kr (W.S.); jeonghwan.lee@khu.ac.kr (J.L.) \\ * Correspondence: kunok.chang@khu.ac.kr; Tel.: +82-31-201-2783
}

Received: 7 August 2020; Accepted: 8 September 2020; Published: 9 September 2020

check for updates

\begin{abstract}
The effects of inhomogeneous elasticity and dislocation on the microstructure evolution of $\alpha^{\prime}$ precipitate in a Fe-Cr system was investigated using a Computer Coupling of Phase Diagrams and Thermochemistry (CALPHAD)-type free energy incorporated phase-field method. In order to simulate the precipitation behavior by phase-field modeling in consideration of inhomogeneous elasticity, a Multiphysics Object-Oriented Simulation Environment (MOOSE) framework was used, which makes it easy to use powerful numerical means such as parallel computing and finite element method (FEM) solver. The effect of inhomogeneous elasticity due to the compositional inhomogeneity or the presence of dislocations affects the thermodynamic properties of the system was investigated, such as the lowest $\mathrm{Cr}$ concentration at which spinodal decomposition occurs. The effect of inhomogeneous elasticity on phase separation kinetics is also studied. Finally, we analyzed how inhomogeneous elasticity caused by compositional fluctuation or dislocation affects microstructure characteristics such as ratio between maximum precipitate size with respect to the average on early stage and later stage, respectively.
\end{abstract}

Keywords: spinodal decomposition; phase-field method; MOOSE framework

\section{Introduction}

$\alpha^{\prime}$ precipitate hardening in a Fe-Cr system is an important research subject in terms of maintaining the mechanical integrity of ferritic steels [1-7]. Precipitation can occur through the spinodal decomposition and the nucleation and growth mechanism, and researchers reported that both mechanisms are involved in the precipitation of $\alpha^{\prime}[2,3]$. The effects of inhomogeneous elasticity and dislocation on Fe-Cr spinodal decomposition have been investigated [6] using the phase-field method; however, the evaluation of the minimum $\mathrm{Cr}$ concentration at which spinodal decomposition occurs was not performed, and the quantitative microstructure analysis was also limited. In this study, we analyzed the effect of the dislocation on the kinetics of the spinodal decomposition and, in particular, the effect on the lowest nominal composition of $\mathrm{Cr}$ for the spinodal decomposition. Through this study, we improved our systematic understanding regarding $\alpha^{\prime}$ nucleation in the presence of the dislocation.

The role of the elasticity on the spinodal decomposition was analyzed using analytic approaches [8,9]. For cubic crystals [8], Cahn claimed that coherent elastic interaction increased the free energy, and therefore inhibited the spinodal decomposition. On the other hand, Eshelby predicted that spinodal decomposition would be accelerated when elastic interaction was considered based on the theory of continuum elastic mechanics [9]. At first glance, this appears to be a conflicting argument; however, Eshelby's predictions describe the rate at which spinodal decomposition occurs and Cahn's 
theoretical study describes the thermodynamic conditions associated with spinodal decomposition. With the phase-field modeling, $\mathrm{Li}$ et al. verified that elasticity accelerates the spinodal decomposition, which shows the consistency with Eshelby's prediction [6]. In this study, we investigated whether the presence of elasticity inhibited the initiation of spinodal decomposition, as Cahn suggested [8].

All simulations were performed at the temperature $T=535 \mathrm{~K}$ [6] using the phase-field method. The phase-field method is a method that has been actively utilized and verified to simulate the $\alpha^{\prime}$ phase precipitation in the Fe-Cr system $[6,10,11]$ with the free energy assessed using the CALPHAD approach [12]. We conducted a set of simulations by utilizing the Multiphysics Object Oriented Simulation Environment (MOOSE) framework, which can easily integrate kernels, such as the finite element method (FEM)-based phase-field and tensor mechanics for elasticity and nucleation. The Fe-Cr microstructure modeling solver that combines the semi-implicit Fourier-spectral method with GPU acceleration was previously developed by the authors of this paper [11]. To consider the elastic effect and nucleation process, we decided to utilize the MOOSE framework, which provides a proven module. From the perspective of solving only the Cahn-Hilliard equation [13], the FEM method, which consumes intensive time to construct the mesh, takes an order of magnitude longer time than the spectral method. However, the constructed mesh is reused in the tensor mechanics module; therefore, the performance gap is greatly reduced when we consider the effect of the dislocation.

\section{CALPHAD-Based Phase-Field Method}

\subsection{Cahn-Hilliard Equation}

The $\mathrm{Cr}$ concentration field will be evolved by solving the following Cahn-Hilliard equation [13,14],

$$
\begin{gathered}
\frac{\partial c(\mathbf{r}, t)}{\partial t}=\nabla \cdot\left[M(\mathbf{r}, t) \cdot \nabla\left(\frac{\delta F(\mathbf{r}, t)}{\delta c}\right)\right] \\
F(\mathbf{r}, t)=\int_{V}\left(\left[f(c)+\frac{1}{2} \kappa(\nabla c)^{2}\right]+f_{e}(c, \mathbf{r})\right) \mathrm{dV} .
\end{gathered}
$$

The molar free energy $F(r, t)$ in Equation (1) is given by Equation (2), and $M(r, t)$ is the mobility of the diffusion species. The mobility was assumed as a constant, and the value was set to 1.0 in this study. Moreover, $\kappa$ is the gradient coefficient.

\subsection{Free Energy of $\mathrm{Fe}-\mathrm{Cr}$ System with Dislocation}

The regular-solution type chemical free energy $f(c)$ in Equation (2) is obtained from [15]

$$
f(c)=(1-c) G_{F e}^{0}+c G_{C r}^{0}+L_{F e C r} c(1-c)+R T(c \ln c+(1-c) \ln (1-c)](\mathrm{J} / \mathrm{mol})
$$

where $c$ is the composition of $\mathrm{Cr}$, and $G_{F e}^{0}$ and $G_{C r}^{0}$ are the molar Gibbs free energies for the elements Fe and $\mathrm{Cr}$, respectively. $L_{\mathrm{FeCr}}$ is the interaction parameter of $\mathrm{Fe}$ and $\mathrm{Cr}$. The detailed numbers are written in $[11,15]$. We set the temperature as $T=535 \mathrm{~K}$. Moreover, we plot the phase diagram in Fe-Cr system in Figure 1 using the FactSage thermochemical software and the FSstel database, which is the FactSage steel alloy database [16].

We determined the elastic energy density $f_{e}(c, \mathbf{r})$ using Equation (2). We implemented Khachaturyan's strain interpolation scheme (KHS) [17]. The stiffness tensor and misfit strain were obtained by the interpolation and create a global stiffness tensor, which is a linear combination of end members $\mathrm{Cr}$ and $\mathrm{Fe}$,

$$
C_{i j k l}^{K H S}(c)=C_{i j k l, C r} \times c+C_{i j k l, F e} \times(1-c)
$$

and the global strain is

$$
\epsilon_{i j}^{K H S}(\mathbf{r})=\epsilon_{i j}^{t o t}(\mathbf{r})-c \times \epsilon_{i j, \alpha^{\prime}}^{\circ}-\epsilon_{i j, d}^{\circ}(\mathbf{r})
$$


where $\epsilon_{i j}^{\text {tot }}(\mathbf{r})$ is the total strain, $\epsilon_{i j, \alpha^{\prime}}^{\circ}$ is the eigenstrain of $\alpha^{\prime}$ phase, and $\epsilon_{i j, d}^{\circ}(\mathbf{r})$ is the eigenstrain induced by the dislocation. The elastic energy density is given by

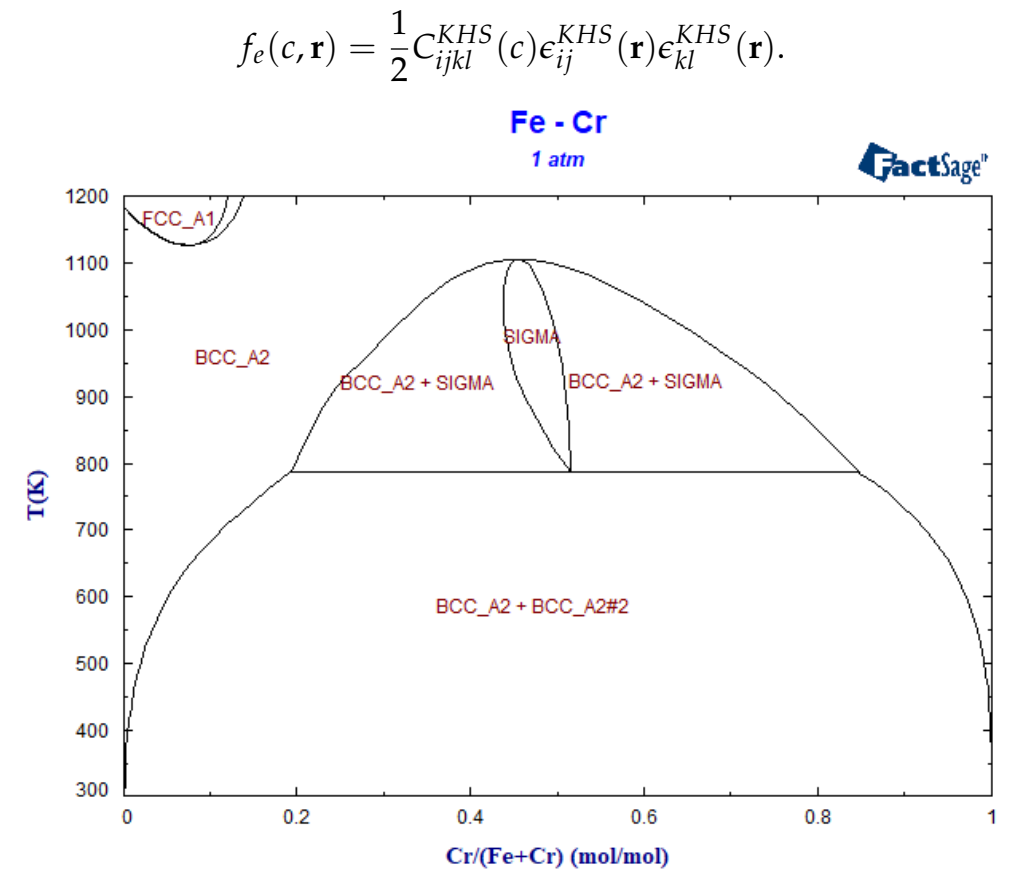

Figure 1. Plot of the phase diagram in Fe-Cr system using FactSage thermochmical software.

The mechanical equilibrium equation was solved to obtain the strain-stress state as below.

$$
\nabla \cdot \sigma_{i j}(\mathbf{r})=0
$$

The tensor-mechanics kernels was utilized in the MOOSE framework to solve Equation (5).

\subsection{Computational Details}

First, we normalized the free energy in Equation (3) by $R T$ for computational efficiency.

$$
f^{*}(c)=\frac{f(c)}{R T}
$$

Two options were considered for the normalized strain tensor (1) written in Table 1A [6], and (2) written in Table 1B. The stiffness tensor of $\mathrm{Fe}$ and $\mathrm{Cr}$ in Table $1 \mathrm{~A}$ has a value at room temperature, and the values in Table $1 \mathrm{~B}$ are dependent on the temperature. The stiffness tensor of Fe in Table $1 \mathrm{~B}$ at $535 \mathrm{~K}$ was calculated as quadratic approximation by extrapolation. As the stiffness of $\mathrm{Cr}$ has insufficient data to extrapolation, it was assumed to be the values at 500K. To examine how much the microstructure varies depending on which of the two different stiffness tensors in Table 1 is chosen, we evaluated microstructure of Fe-21Cr system with two different sets of stiffness tensor and the results of the average $\alpha^{\prime}$ radius and the number of $\alpha^{\prime}$ precipitation were plotted in Figures 2 and 3. As no significant difference was found in the microstructure characteristics in the two results, it was chosen to use a value at room temperature in Table $1 \mathrm{~A}$ rather than the value calculated by extrapolation, even if there was a dependence on temperature in the stiffness. 
Table 1. The normalized stiffness tensor of Fe and $\mathrm{Cr}$ at 535K in Voigt notation [18-20].

\begin{tabular}{ccc}
\hline \multicolumn{3}{c}{$\mathbf{A}$} \\
\hline & $\mathbf{F e}$ & $\mathbf{C r}$ \\
\hline$C_{11}^{*}$ & 233.10 & 350.00 \\
$C_{12}^{*}$ & 135.44 & 67.80 \\
$C_{44}^{*}$ & 178.30 & 100.80 \\
\hline \multicolumn{3}{c}{$\mathbf{B}$} \\
\hline \multicolumn{3}{c}{$\mathbf{F e}$} \\
\hline$C_{11}^{*}$ & $\mathbf{C r}$ \\
$C_{12}^{*}$ & 130.70 & 76.94 \\
$C_{44}^{*}$ & 110.72 & 98.70 \\
\hline
\end{tabular}

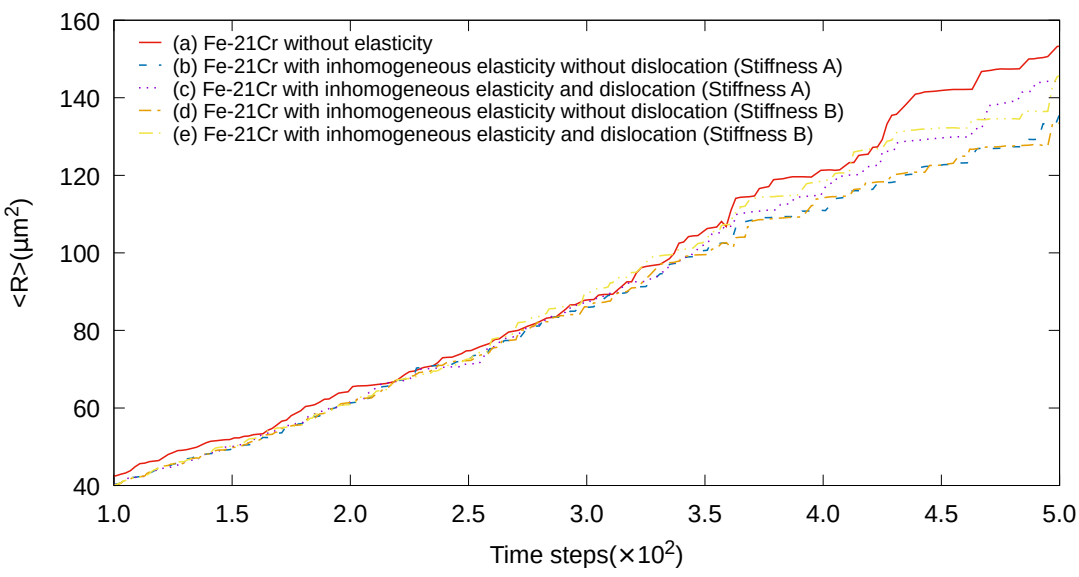

Figure 2. The average $\alpha^{\prime}$ radius $(<R>)$ with respect to time for the cases of (a) without elasticity, (b) with inhomogeneous elasticity having the stiffness in Table 1A without dislocation, (c) with inhomogeneous elasticity having the stiffness in Table $1 \mathrm{~A}$ and dislocation, (d) with inhomogeneous elasticity having the stiffness in Table 1B without dislocation, and (e) with inhomogeneous elasticity having the stiffness in Table 1B with dislocation.

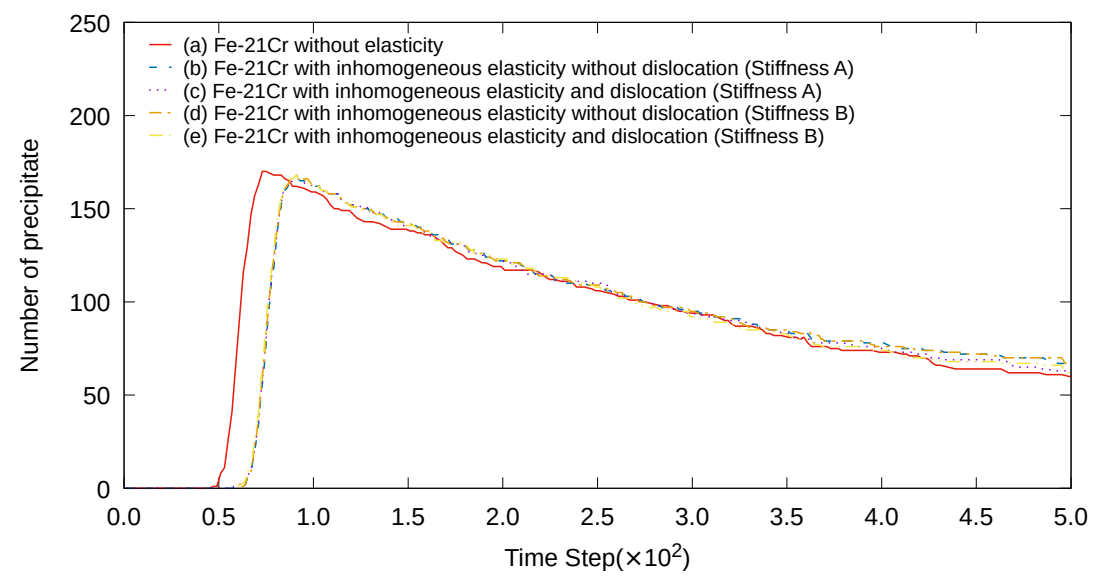

Figure 3. The number of $\alpha^{\prime}$ precipitations with respect to the time for the case of (a) without elasticity, (b) with inhomogeneous elasticity having the stiffness in Table 1A without dislocation, (c) with inhomogeneous elasticity having the stiffness in Table 1A and dislocation, (d) with inhomogeneous elasticity having the stiffness in Table 1B without dislocation, and (e) with inhomogeneous elasticity having the stiffness in Table 1B with dislocation. 
We used $\kappa=4.25$ in Equation (2). The eigenstrain of $\alpha^{\prime}$ phase $\epsilon_{i j, \alpha^{\prime}}^{\circ}$ in Equation (4) is 0.006 when $i=j[6] . \epsilon_{i j, d}^{\circ}(\mathbf{r})$ in Equation (4) is evaluated by the authors of [21].

$$
\epsilon_{i j, d}^{\circ}(\mathbf{r})=\mathbf{b}(\mathbf{r}) \otimes \mathbf{n}=\frac{b_{i}(\mathbf{r}) n_{j}+b_{j}(\mathbf{r}) n_{i}}{2 d}
$$

In 2D, we assume the Burger's vector $\mathbf{b}(\mathbf{r})=\frac{a_{0}}{2}[111]$ on the line connecting two dislocations on the (110) slip plane, where $a_{0}$ is the lattice parameter [21]. The lattice is rotated around the $x$-axis [11̄0], $y$-axis [111], and $z$-axis [1̄12]; therefore, all terms without shear terms become 0 [21]. We set $\epsilon_{12, d}^{\circ}(\mathbf{r})=0.005$ within the dislocation dipole in this study. We set $\Delta x=\Delta y=1.0 \times 10^{-8} \mathrm{~m}$, which were implemented by $\Delta x^{*}=\Delta y^{*}=1.0$ after normalization. The total number of meshes in the 2 D system was $256 \times 256$.

\section{Results and Discussions}

The Effect of Elasticity and Dislocation on $\alpha^{\prime}$ Phase Precipitation Induced by the Spinodal Decomposition Mechanism

We examined multiple compositions within the range of $\mathrm{Fe}-14 \mathrm{Cr}$ to $\mathrm{Fe}-21 \mathrm{Cr}$ to investigate the role of dislocation on $\alpha^{\prime}$ precipitate nucleation through the spinodal decomposition. To examine the role of the inhomogeneous elasticity and the dislocation, we performed simulations (1) without the $f_{e}$ term in Equation (3) (no elasticity case), (2) with the $f_{e}$ term without dislocation (with elasticity only arising from the concentration inhomogeneity considered), and (3) with the $f_{e}$ term with the dislocation.

Typically, the concentration of $\mathrm{Cr}$ in Fe-Cr steel is expressed as wt\%; however, in this study, the value was set in terms of at $\%$ in the simulation. For example, in the Fe- $13 \mathrm{Cr}$ system, we set the initial average composition of $\mathrm{Cr}$ was 13.86 at $\%$. The initial $\mathrm{Cr}$ concentration spanned from 13.36 at $\%$ to 14.36 at $\%$. The range of the fluctuation was \pm 0.5 at $\%$ for all the cases. First, we found that the presence of the inhomogeneous concentration did not alter the minimum $\mathrm{Cr}$ average concentration for the spinodal decomposition of $\mathrm{Fe}-16.7 \mathrm{Cr}$; however, it altered the kinetics significantly. We observed the $\alpha^{\prime}$ precipitate from $t=14,300$ when inhomogeneous elasticity is not incorporated; on the other hand, we observed $\alpha^{\prime}$ precipitate from $t=1732$ when we considered inhomogeneous elasticity. This observation is consistent with the predictions made by Eshelby [9].

We also found that presence of the dislocation lowered the minimum $\mathrm{Cr}$ concentration for spinodal decomposition. As shown in Figure 4, we found that the $\alpha^{\prime}$ phase nucleated even in Fe-16.4Cr. As the $\mathrm{Cr}$ concentration increased in the vicinity of the dislocation when compared to the nominal composition, dislocation is a suitable location for the $\alpha^{\prime}$ nucleation as experimentally observed [22]. To confirm these findings, we performed four different sets of simulation with different random seeds.

We also analyzed the role of the inhomogeneous elasticity and dislocation on the microstructural evolution of Fe-21Cr in Figure 5. As shown in Figures 2 and 6, when elasticity was not considered, the microstructure evolution was the fastest. When elasticity only due to the compositional inhomogeneity was considered, the average precipitate size increased and the increased rate of the $\alpha^{\prime}$ phase fraction were the slowest among the three cases.

In summary, when elasticity was not considered, the system approached equilibrium the most rapidly. However, even if elasticity was considered, if the effects of structural inhomogeneity, such as dislocation, were considered simultaneously, the growth rate of the precipitate was faster in the case of the absence of the dislocation with consideration of the elasticity. At $t=70$, as shown in Figure $7 \mathrm{f}$, there was $\mathrm{Cr}$ concentration partitioning when a dislocation was present in the particle; therefore, a relatively large $\alpha^{\prime}$ precipitate existed in the vicinity of the dislocation.

In Figure 5, we observed overshootings in the $R_{m} /<R>$ plot at early stages for all three cases, where $R_{m}$ represents the maximum radius of the $\alpha^{\prime}$ phase and $<R>$ is the average radius of the $\alpha^{\prime}$ precipitate. In the presence of elasticity only arising from compositional inhomogeneity, transient overshooting was lower than case of the absence of the elasticity. However, with the presence of 
dislocation, the largest overshooting existed at approximately $t=70$, where $R_{m} /<R>$ exceeded 3.5. This indicates that the largest relative $\alpha^{\prime}$ precipitate existed with respect to the average value among all situations.

As time proceeded, $<R>$ increased and, after a certain time (after $t=470$ in Figure 5 ), $R_{m} /<R>$ demonstrated the maximum value with the presence of inhomogeneous elasticity without any dislocation. When a dislocation was present in the system, the morphology of the $\alpha^{\prime}$ precipitate deviated from the circular shape as shown in Figure 4; thereby, the driving force for the coarsening reduced [23]. Consequently, the original largest precipitates in the vicinity of the dislocation grew relatively slowly in the late stage, and the value of $R_{m} /<R>$ became smaller compared to the case of the presence of dislocation.

According to the analytic theory developed by Kawasaki and Enomoto [24], the size distribution of coarsened particles becomes wide when a repulsive elastic field is applied. As the eigenstrain is proportional to the $\mathrm{Cr}$ composition, we determined that repulsive elastic fields were generated between the $\mathrm{Cr}$ rich phases, and accordingly, the $R_{m} /<R>$ value was found to be larger in the presence of elasticity due to the concentration inhomogeneity without dislocation in the later stage.

In the following Figures 7 and 8, the $\alpha^{\prime}$ phase nucleation was more progressed in the $\mathrm{Fe}-21 \mathrm{Cr}$ system without elasticity (a) than in the cases with inhomogeneous elasticity at $t=70(\mathrm{~b}, \mathrm{c})$. The Fe-Cr21 system with inhomogeneous elasticity and the dislocation showed more active $\alpha^{\prime}$ nucleation in the vicinity of the dislocation than at other sites. In this study, the effect of inhomogeneous elasticity upon precipitation of precipitates by spinodal decomposition was evaluated, but, in fact, precipitates can also be initiated and evolved by nucleation and growth mechanisms [25]. In fact, the authors of [25] state that the $\alpha^{\prime}$ precipitation mechanism changes on $\mathrm{Fe}-\mathrm{Cr}$ at $28 \mathrm{Cr}$. It is necessary to quantitatively analyze the effect of inhomogeneous elasticity when $\alpha^{\prime}$ phase is precipitated by the nucleation and growth mechanism. A more reliable explanation on the effect of elasticity on nucleation frequency or critical nuclei size is necessary to quantitatively predict phase separation kinetics induced by nucleation and growth mechanism.

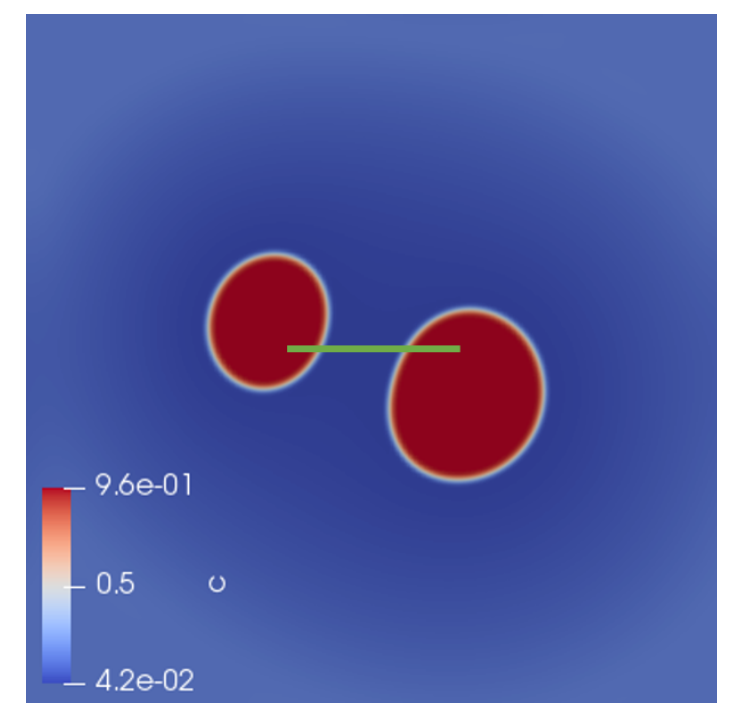

Figure 4. The $\alpha^{\prime}$ phase is nucleated in the Fe-16.4Cr system in the presence of the dislocation. The system size was $256 \times 256 \mu \mathrm{m}^{2}$. The green horizontal line in the middle represents the line connecting dislocations. The microstructures were visualized by $\mathrm{Cr}$ composition. 


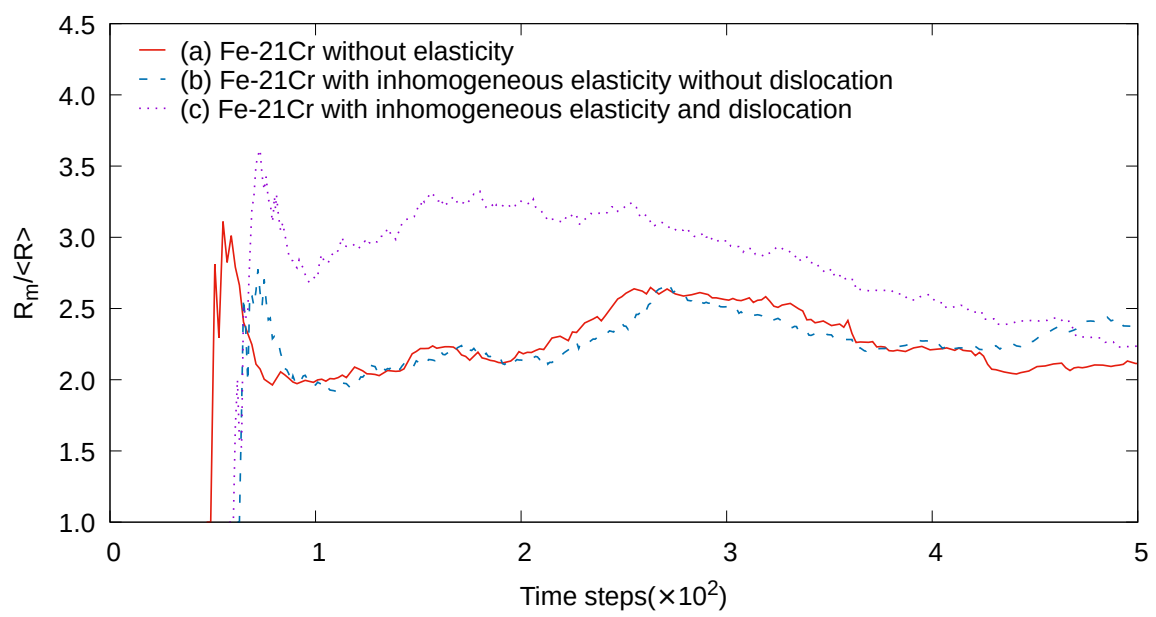

Figure 5. $R_{m} /<R>$ with respect to time for the cases of (a) without elasticity, (b) with inhomogeneous elasticity without dislocation, and (c) with inhomogeneous elasticity and dislocation, where $R_{m}$ is the maximum radius of the $\alpha^{\prime}$ precipitate and $<R>$ is the average radius of it. We performed four different sets of simulations to evaluate $R_{m}$ and $<R>$ for enhanced statistical rigor of the analysis. The system size was $256 \times 256 \mu \mathrm{m}^{2}$. The composition of the system is Fe-21Cr.

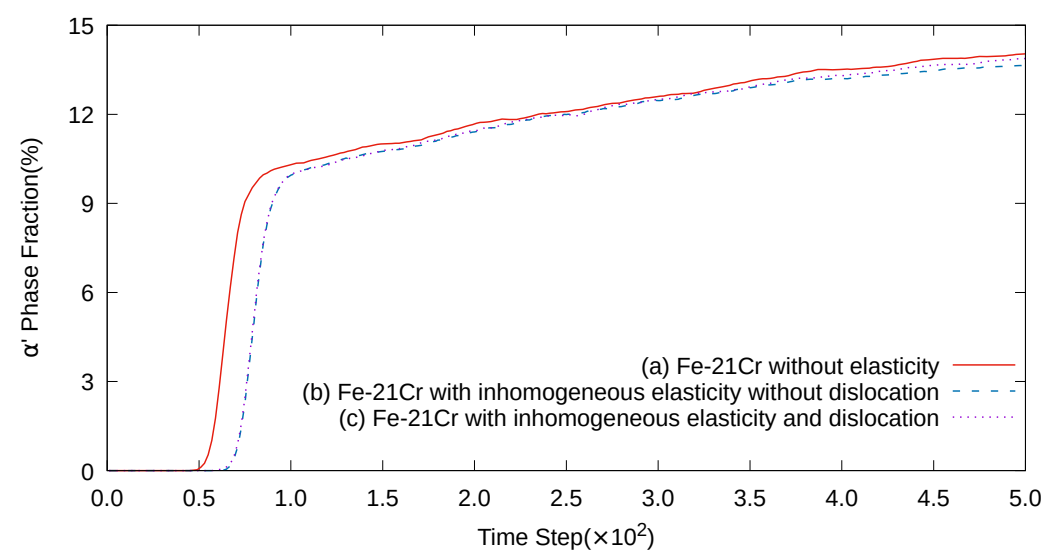

Figure 6. The $\alpha^{\prime}$ phase fraction with respect to the time for the cases of (a) without elasticity, (b) with inhomogeneous elasticity without dislocation, and (c) with inhomogeneous elasticity and dislocation.

Cr segregation in the vicinity of dislocation (loop) has been consistently observed experimentally [22,26,27]; however, no studies have been found showing evidence that the segregation leads to $\alpha^{\prime}$ precipitation. It is possible to estimate that a locally high $\mathrm{Cr}$ concentration near the microstructure inhomogeneity, such as dislocation, will help in the formation of $\mathrm{Cr}$-rich precipitate, and this study showed the anticipation is the case using the phase-field simulation. Studies on $\alpha^{\prime}$ behavior at the early stage of the phase separation in the Fe-Cr system have been conducted experimentally with relatively high $\mathrm{Cr}$ compositions, such as $\mathrm{Fe}-32 \mathrm{wt} \% \mathrm{Cr}[5,28], \mathrm{Fe}-36 \mathrm{wt} \% \mathrm{Cr}$ [29] and $\mathrm{Fe}-25.28 \mathrm{wt} \% \mathrm{Cr}$, and $\mathrm{Fe}-30.42 \mathrm{wt} \% \mathrm{Cr}$ and $\mathrm{Fe}-36.10 \mathrm{wt} \% \mathrm{Cr}$ [30]. Their concentration ranges are clearly regions in which spinodal decomposition occurs, and the above studies [5,28-30] are valuable studies that help to understand the early-stage microstructural behavior when the phase separation occurs by spinodal decomposition. Relatively recently, there has been a report that $\alpha^{\prime}$ is precipitated even in Fe- 9 at\% $\mathrm{Cr}$ during neutron irradiation. In this case, one cannot confidently claim that $\alpha^{\prime}$ precipitation is nucleated by spinodal decomposition, if no other effects are included other than the 
composition. From this experimental observation, we hypothesized that the dislocation loop generated by neutron irradiation caused $\mathrm{Cr}$ partitioning to lower the lowest average $\mathrm{Cr}$ concentration at which phase separation occurred, and phase-field modeling was used to verify it. Our results found that the dislocation lowered the lowest mean $\mathrm{Cr}$ concentration at which spinodal occurs, even though the effect is relatively marginal. Additionally, it was found that the incubation time required for $\alpha^{\prime}$ to precipitate was significantly reduced for Fe- $16.4 \mathrm{wt} \% \mathrm{Cr}$ by $\mathrm{Cr}$ partitioning by the dislocation. Moreover, we show that the model for Fe-Cr microstructure evolution with elastic effect proposed in [6] can be performed without advanced programming skill by utilizing MOOSE framework.

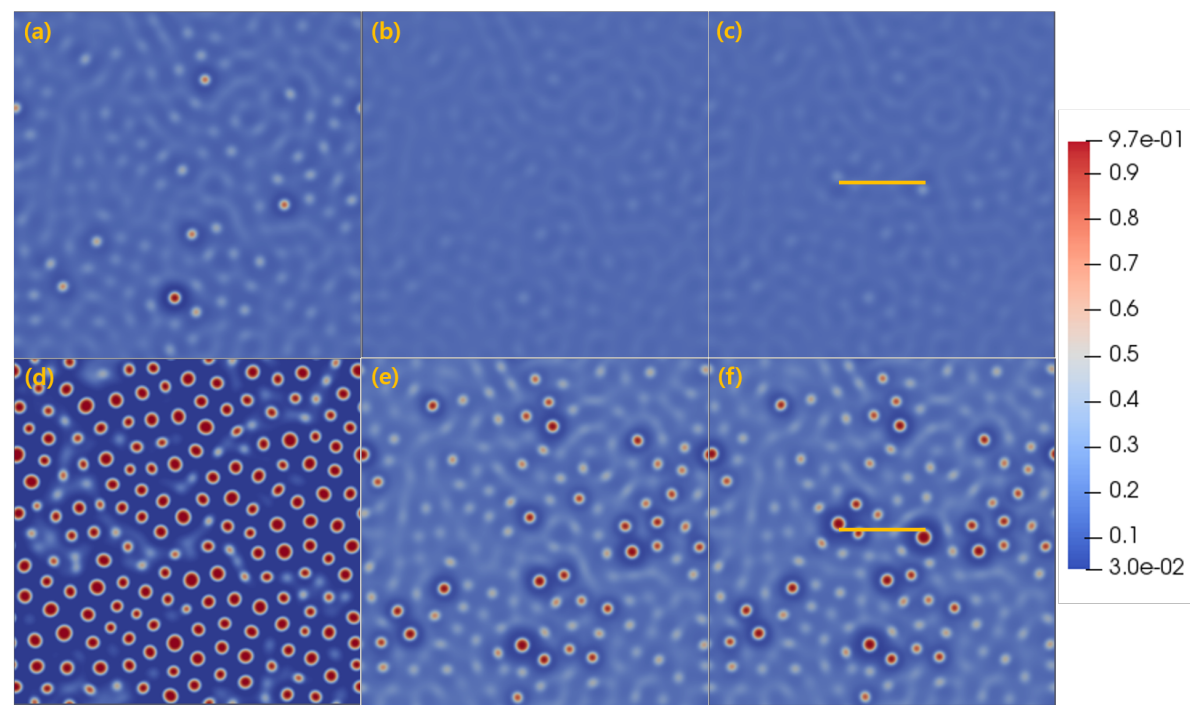

Figure 7. Microstructures of the Fe-21Cr system (a) without elasticity at $t=50$, (b) with inhomogeneous elasticity without dislocation at $t=50$, (c) with inhomogeneous elasticity and dislocation at $t=50$, (d) without elasticity at $t=70$, (e) with inhomogeneous elasticity without dislocation at $t=70$, and (f) with inhomogeneous elasticity and dislocation at $t=70$. The system size was $256 \times 256 \mu \mathrm{m}^{2}$. The microstructures were visualized by $\mathrm{Cr}$ composition.

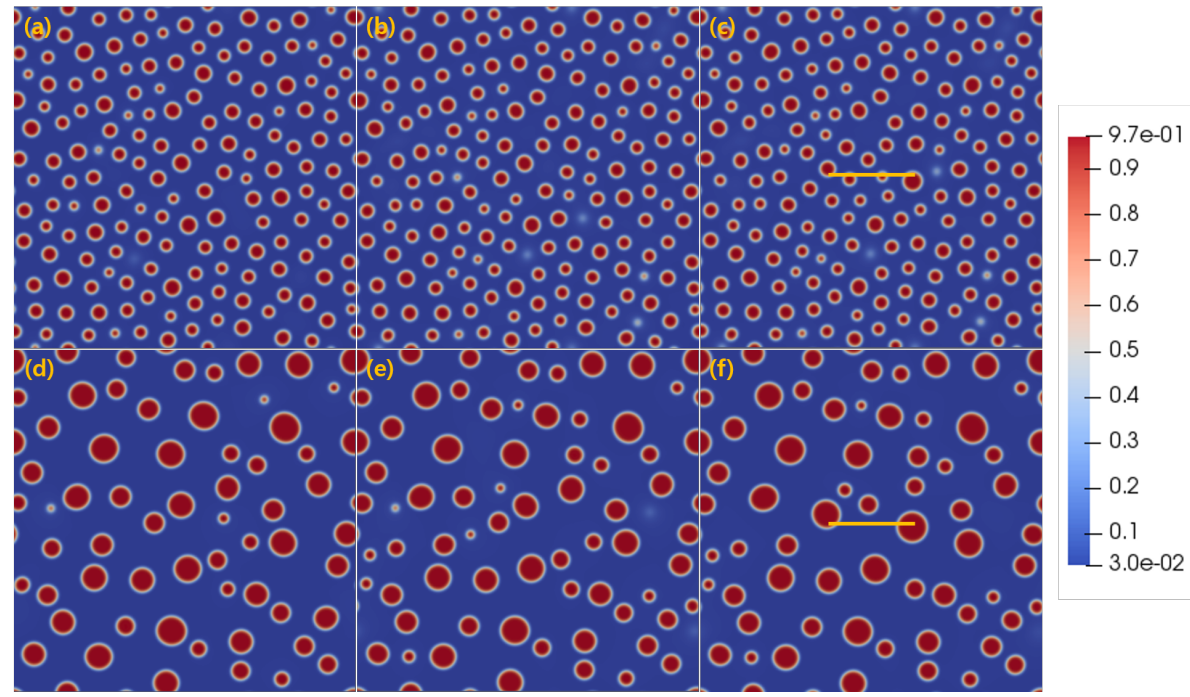

Figure 8. Microstructures of the Fe-21Cr system (a) without elasticity at $t=100$, (b) with inhomogeneous elasticity without dislocation at $t=100$, (c) with inhomogeneous elasticity and dislocation at $t=100$, (d) without elasticity at $t=500$, (e) with inhomogeneous elasticity without dislocation at $t=500$, and (f) with inhomogeneous elasticity and dislocation at $t=500$. The system size was $256 \times 256 \mu \mathrm{m}^{2}$. The microstructures were visualized by $\mathrm{Cr}$ composition. 


\section{Conclusions}

Phase-field simulation using the MOOSE framework has been utilized to study the effect of inhomogeneous elasticity including dislocations on $\alpha^{\prime}$ precipitate nucleation through the spinodal decomposition in Fe-Cr alloy. Our study yields three main findings: We found that inhomogeneous elasticity induced by the compositional inhomogeneity of the $\alpha$ phase did not alter the minimum Cr composition for the spinodal decomposition; however, it altered the kinetics significantly. Moreover, we found that the presence of the dislocation lowered the minimum Cr composition for spinodal decomposition. At the early stage of the phase separation, the relative size of the $\alpha^{\prime}$ precipitate was maximized when a dislocation existed in the system. In the later stage, the relative size of the precipitate showed the maximum size when inhomogeneous elasticity was incorporated without any dislocation.

Author Contributions: Conceptualization, K.C.; methodology, W.S. and J.L.; software, W.S. and J.L.; validation, K.C. and W.S.; formal analysis, W.S.; investigation, W.S.; resources, W.S.; data curation, W.S.; writing-original draft preparation, K.C.; writing-review and editing, K.C.; visualization, W.S.; supervision, K.C.; project administration, K.C.; funding acquisition, K.C. All authors have read and agreed to the published version of the manuscript.

Funding: This work has supported by the National Research Foundation of Korea (NRF) grant funded by the Korea government (MSIT) (NRF-2019M2D2A1A01032534). This work was also supported by the "Human Resources Program in Energy Technology" of the Korea Institute of Energy Technology Evaluation and Planning (KETEP), granted financial resources from the Ministry of Trade, Industry \& Energy, Republic of Korea (No. 20184030202170).

Conflicts of Interest: The authors declare no conflict of interest.

\section{References}

1. Grobner, P. The 885 F (475 C) embrittlement of ferritic stainless steels. Metall. Trans. 1973, 4, 251-260. [CrossRef]

2. Chandra, D.; Schwartz, L. Mössbauer effect study of the $475 \ddagger$ C decomposition of Fe-Cr. Metall. Trans. 1971, 2, 511-519. [CrossRef]

3. Cieślak, J.; Dubiel, S. Nucleation and growth versus spinodal decomposition in Fe-Cr alloys: Mössbauer-effect modelling. J. Alloys Compd. 1998, 269, 208-218. [CrossRef]

4. Solomon, H.; Levinson, L.M. Mössbauer effect study of '475 C embrittlement' of duplex and ferritic stainless steels. Acta Metall. 1978, 26, 429-442. [CrossRef]

5. Miller, M.; Hyde, J.; Hetherington, M.; Cerezo, A.; Smith, G.; Elliott, C. Spinodal decomposition in Fe-Cr alloys: Experimental study at the atomic level and comparison with computer models-I. Introduction and methodology. Acta Metall. Mater. 1995, 43, 3385-3401. [CrossRef]

6. Li, Y.S.; Li, S.X.; Zhang, T.Y. Effect of dislocations on spinodal decomposition in Fe-Cr alloys. J. Nucl. Mater. 2009, 395, 120-130. [CrossRef]

7. Hedström, P.; Baghsheikhi, S.; Liu, P.; Odqvist, J. A phase-field and electron microscopy study of phase separation in Fe-Cr alloys. Mater. Sci. Eng. A 2012, 534, 552-556. [CrossRef]

8. Cahn, J.W. On spinodal decomposition in cubic crystals. Acta Metall. 1962, 10, 179-183. [CrossRef]

9. Eshelby, J. The continuum theory of lattice defects. In Solid State Physics; Elsevier: Amsterdam, The Netherlands, 1956; Volume 3, pp. 79-144.

10. Zhu, L.; Li, Y.; Liu, C.; Chen, S.; Shi, S.; Jin, S. Effect of applied strain on phase separation of Fe-28 at.\% Cr alloy: 3D phase-field simulation. Model. Simul. Mater. Sci. Eng. 2018, 26, 035015. [CrossRef]

11. Lee, J.; Chang, K. Effect of magnetic ordering on the spinodal decomposition of the Fe-Cr system: A GPU-accelerated phase-field study. Comput. Mater. Sci. 2019, 169, 109088. [CrossRef]

12. Dinsdale, A. SGTE data for pure elements. Calphad 1991, 15, 317-425. [CrossRef]

13. Cahn, J.W.; Hilliard, J.E. Free energy of a nonuniform system. I. Interfacial free energy. J. Chem. Phys. 1958, 28, 258-267. [CrossRef]

14. Cahn, J.W. On spinodal decomposition. Acta Metall. 1961, 9, 795-801. [CrossRef]

15. Andersson, J.O.; Sundman, B. Thermodynamic properties of the Cr-Fe system. Calphad 1987, 11, 83-92. [CrossRef] 
16. Bale, C.W.; Chartrand, P.; Degterov, S.; Eriksson, G.; Hack, K.; Mahfoud, R.B.; Melançon, J.; Pelton, A.; Petersen, S. FactSage thermochemical software and databases. Calphad 2002, 26, 189-228. [CrossRef]

17. Khachaturyan, A.G. Theory of Structural Transformations in Solids; Courier Corporation: Mineola, NY, USA, 2013.

18. Newnham, R.E. Properties of Materials: Anisotropy, Symmetry, Structure; Oxford University Press on Demand: Oxford, UK, 2005.

19. Adams, J.J.; Agosta, D.; Leisure, R.; Ledbetter, H. Elastic constants of monocrystal iron from 3 to $500 \mathrm{~K}$. J. Appl. Phys. 2006, 100, 113530. [CrossRef]

20. Bolef, D.T.; De Klerk, J. Anomalies in the elastic constants and thermal expansion of chromium single crystals. Phys. Rev. 1963, 129, 1063. [CrossRef]

21. Biner, S.B. Programming Phase-Field Modeling; Springer: Berlin/Heidelberg, Germany, 2017.

22. Terentyev, D.; Bergner, F.; Osetsky, Y. Cr segregation on dislocation loops enhances hardening in ferritic Fe-Cr alloys. Acta Mater. 2013, 61, 1444-1453. [CrossRef]

23. Porter, D.A.; Easterling, K.E.; Sherif, M. Phase Transformations in Metals and Alloys, (Revised Reprint); CRC Press: Boca Raton, FL, USA, 2009.

24. Kawasaki, K.; Enomoto, Y. Statistical theory of Ostwald ripening with elastic field interaction. Phys. Stat. Mech. Appl. 1988, 150, 463-498. [CrossRef]

25. Li, Y.; Yan, Z.; Zhou, X. Kinetics of initial phase separation and coarsening of nanoscale phase in $\mathrm{Fe}-\mathrm{Cr}$ alloys. J. Nucl. Mater. 2017, 497, 154-160. [CrossRef]

26. Bachhav, M.; Odette, G.R.; Marquis, E.A. $\alpha^{\prime}$ precipitation in neutron-irradiated Fe-Cr alloys. Scr. Mater. 2014, 74, 48-51. [CrossRef]

27. Porollo, S.; Dvoriashin, A.; Vorobyev, A.; Konobeev, Y.V. The microstructure and tensile properties of Fe-Cr alloys after neutron irradiation at $400 \mathrm{C}$ to 5.5-7.1 dpa. J. Nucl. Mater. 1998, 256, 247-253. [CrossRef]

28. Hyde, J.; Miller, M.; Hetherington, M.; Cerezo, A.; Smith, G.; Elliott, C. Spinodal decomposition in Fe-Cr alloys: Experimental study at the atomic level and comparison with computer models-II. Development of domain size and composition amplitude. Acta Metall. Mater. 1995, 43, 3403-3413. [CrossRef]

29. Westraadt, J.; Olivier, E.; Neethling, J.; Hedström, P.; Odqvist, J.; Xu, X.; Steuwer, A. A high-resolution analytical scanning transmission electron microscopy study of the early stages of spinodal decomposition in binary Fe-Cr. Mater. Charact. 2015, 109, 216-221. [CrossRef]

30. Zhou, J.; Odqvist, J.; Thuvander, M.; Hedström, P. Quantitative evaluation of spinodal decomposition in Fe-Cr by atom probe tomography and radial distribution function analysis. Microsc. Microanal. 2013, 19, 665-675. [CrossRef]

(C) 2020 by the authors. Licensee MDPI, Basel, Switzerland. This article is an open access article distributed under the terms and conditions of the Creative Commons Attribution (CC BY) license (http://creativecommons.org/licenses/by/4.0/). 